\title{
Significado y obstáculos para la labor innovadora en una escuela
}

\author{
Víctor Julio Baltodano Zúñiga \\ Ana Rita Badilla Alvarado ${ }^{6}$
}

\section{RESUMEN}

El presente artículo aborda el significado y factores que obstaculizan la innovación educativa en una escuela, en este caso la Escuela Presbítero José Daniel Carmona Briceño de Ciudad Carmona, Nandayure, Guanacaste. Dentro de los principales factores se apuntan: disponibilidad de tiempo, problemas presupuestarios, desgaste físico y emocional, capacitación, pocá o nula sistematización, horarios con estructuración poco lógica, falta de apoyo por parte de las autoridades y, en términos generales, falta de una cultura dentro de la educación primaria para innovar.

Se reconoce la importancia de la innovación educativa como elemento fundamental que genera un aprendizaje significativo; pero a la vez la dificultad que se tiene para que esta se lleve a la práctica. En el caso de los niños, a pesar de tener una imagen muy positiva de sus maestras, apuntan que lo excesivamente teórico de las clases que reciben los desmotivan. Por lo

\footnotetext{
Acadérnico de la Sede Regional Chorotega de la U.N.A y de la Sede de Guanacaste. UCR tiene varias publicaciones en revistas nacionales.

Académica de la Sede Regional Chorotega de la U.N.A. Tiene una publicación en la Revise Inter Sedes de la Universidad de Costa Rica.
} 
tanto, es urgente implementar acciones que conduzcan a clases más atractivas, e innovadoras a la vez, por parte de los docentes.

\section{SUMMARY}

The present article identify the meaning and factors against educative innovation in elementary school; in this case the Presbítero José Daniel Carmona Briceño Elementary School from Nandayure, Guanacaste. Among the main factors of this problem are the availability of time, economical problems, excessive physical and emotional work, lack of training and systematization, lack of logic structuration on schedule, inadequate support by the principal authorities and in general terms lack of culture in primary school about innovation. Educative innovation is a fundamental element for the significant learning of students; nevertheless, it is very difficult to put it on practice because teachers do not have the enough knowledge about it. Most of the time children have a positive imagine about their teachers but the excess of teorical classes produce absence of motivation and the learning process become to decrease. It is for that reason, emerge the necessity of applying new actions in order to teacher creative dynamice, and attractive classes for improving the significant learning in the student.

\section{PALABRAS CLAVES}

Educación primaria, innovación,

\section{KEYS WORDS}

Elementary school, innovation.

\section{ASPECTOS INTRODUCTORIOS}

\section{La Escuela Presbítero José Daniel Carmona Briceño} se encuentra ubicada en el cantón de Nandayure el cual es el noveno de la provincia de Guanacaste. Pertenece al circuito 07 de la Dirección Regional de Enseñanza de Nicoya. La escuela actualmente ostenta la categoría de Dirección 3 y tiene una matrícula de 350 estudiantes. Cuenta con 24 funcionarios entre docentes y administrativos. 
En cuanto a la metodología utilizada en la presente investigación, se puede apuntar que esta tiene un enfoque cualitativo y es de tipo fenomenológico, por lo que trata de describir e interpretar las situaciones tal como se presentan por parte de la población investigada. Cada individuo construye su mundo y le da significado a sus acciones, por lo tanto, sus acciones vienen condicionadas por esa experiencia previa que durante toda su vida ha ido creando. De ese significado que los actores le dan a las innovaciones educativas se extrajeron conclusiones importantes para conocer el porqué de las acciones.

La investigación se realizó tomando en cuenta la opinión de dieciséis niños escogidos ocho del I ciclo y ocho del II Ciclo. Además, estudió las cuatro docentes de esos ciclos así como al Director de la Escuela. Para la recopilación de la información se elaboraron varios instrumentos que involucraban la observación directa, la entrevista a profundidad y las historias de vida.

\section{ELEMENTOS TEÓRICOS QUE SUSTENTAN LA PRACTICA PEDAGÓGICA Y LA INNOVACIÓN EDUCATIVA}

\section{EL CONSTRUCTIVISMO}

Pretende explicar el origen del conocimiento tomando en cuenta que este no se adquiere, sino que cada individuo lo crea o lo recrea en su mente. Dos son los principales exponentes de esta corriente: Jean Piaget y Lev Vygostki. Para Piaget, el conocimiento "es producto de las interacciones entre la persona que aprende y los objetos de la realidad que trata de aprehender (Pérez, 2000, p.20). Por lo tanto, a partir de este interaccionismo se construye el conocimiento. Para la práctica pedagógica lo anterior es importante ya que: 
Los niños en la edad escolar (operaciones concretas) construyen sus conceptos derivándolos directamente en relación con los objetos concretos; de aquí la importancia de usar los procesos de enseñanza y aprendizaje, materiales físicos y concretos, situaciones vivenciales, ejercicios prácticos y, menos las charlas, las exposiciones, en fin el verbalismo. (Pérez y otros, 2000:25)

De lo anterior se deduce que el maestro debe ser creativo e innovador dentro del aula, con el objetivo de hacer más atractivo y efectivo el aprendizaje del niño. Las innovaciones vivenciales, participativas y prácticas facilitan la apropiación del conocimiento; es decir, conceptos, procedimientos y actitudes, por parte del educando. Esta adquisición se da mediante un proceso que tiene etapas o fases: asimilación, acomodación y la equilibración. La asimilación es entendida como la incorporación de un elemento exterior a un esquema de conocimiento ya desarrollado por la persona. O sea el sujeto recoge la nueva información y la adapta a la que ya conoce. La acomodación consiste en la necesidad de digerir la realidad tomando en cuenta las particularidades propias de los elementos que ese debe asimilar. La equilibración es un mecanismo de ajuste al ambiente, producto de las fases de acomodación y asimilación. Se podría decir que existe auténtica apropiación de los conocimientos solo cuando hay equilibración (Pérez y otros, 2000: p29).

Sintetizando, se puede anotar que Piaget brindó grandes aportes a la educación y al aprendizaje, por su gran repercusión en la didáctica. Primero, el reconocimiento de la importancia de las actividades que realiza el sujeto para aprender. Con esto se puede decir que nadie aprende por otro. Por lo tanto, en el momento de planear el proceso de 
aprendizaje es primordial pensar en las actividades que realizan las personas que aprenden. Segundo, se debe tener respeto por la experiencia previa del estudiante, tanto en relación con la edad como con la experiencia vivida, este principio se recupera con los estadios o períodos de desarrollo.

El segundo pensador en importancia en el constructivismo es el ruso Lev Vygostki. Se considera que el constructivismo social es una de las corrientes psicológicas que en la actualidad tiene mayor vigencia sobre la educación. Su propuesta toma cada día mayor valor. "El constructivismo Vygostkiano se caracteriza fundamentalmente por concebir el desarrollo del pensamiento y la conciencia como un proceso de carácter socio-histórico-cultural" (Pérez y otros: 2000, p.34). En otras palabras, la mente del ser humano es una mente social. Los proceso psicológicos específicamente humanos, tales como la percepción, memoria, razonamiento y resolución de problemas, son el resultado de la internalización de las relaciones sociales tales como se dan en una determinada cultura. Desde esta perspectiva, el enfoque de este pensador exige una práctica pedagógica dinámica, activa, constructiva y colaborativa, en este sentido, el autor explica la apropiación del conocimiento como el paso de una zona de desarrollo real en la que se encuentra la persona que aprende a una zona de desarrollo próximo.

\section{EL CONCEPTO DE INNOVACION}

Un concepto de innovación relacionado con la educación es el que ofrece Skatkin (Murillo y otras, 1996: 5). Este autor la define como:

La práctica pedagógica innovadora, es decir, aquella que contiene elementos de búsqueda creadora, de novedad y originalidad, aquella en que el maestro crea 
métodos, procedimientos. Si este tipo de experiencia es especialmente valiosa porque descubre nuevas vías en la práctica escolar y enriquece la teoría pedagógica.

Sin embargo, un concepto mucho más elaborado y contextualizado al aula es el concepto de innovación didáctica emergente proporcionado por Libedinsky (2001:60):

Las innovaciones didácticas emergentes son propuestas de enseñanza generadas por docentes de aula, caracterizadas por la ruptura y oposición con prácticas vigentes consolidadas, profundamente ensambladas con el contenido curricular disciplinar, y que atienden tanto a los intereses culturales de los docentes que las diseñan y lideran, como a los intereses culturales de sus estudiantes.

La autora apunta que la innovación debe responder a una concepción de ruptura con una propuesta didáctica preexistente, además, explica que debe ser una ruptura a partir de la cual se pueda marcar un antes y un después. Por lo tanto, Libedinsky (2001:59) argumenta que:

Se trata de innovaciones emergentes, no institucionales, ya que son los autores quienes las conciben y ellos mismos quienes experimentan sus efectos. Son los docentes quienes producen perturbaciones en su propia práctica cotidiana de manera voluntaria. Es un proceso que se formula desde el interior, desde la historia cultural y generacional de sus autores. Es una forma prudente, en el buen sentido pedagógico, de mejorar la enseñanza, y supone cambios de valores sutiles en la cultura institucional. 
De acuerdo con la idea implícita que encierra este texto, el proceso innovador al comienzo tiene mucha exigencia y agota al maestro sin experiencia; pero luego, si hay un verdadero interés, responsabilidad social y profesionalismo, se convierte en un quehacer cotidiano y permanente para las personas y las instituciones. Y aún más, cuando el proceso innovador nace de él mismo.

\section{SIGNIFICADO DE LA INNOVACIÓN DESDE LA PERSPECTIVA DE LOS EDUCANDOS}

Los estudiantes muestran dificultades al conceptualizar el término "innovación"; sin embargo, recalcan que aprender jugando, hacer o crear, y aprovechar algunos materiales de desecho para la elaboración de otros trabajos son parte de una innovación. Se sienten muy motivados con las materias especiales como música, religión, inglés, hogar y educación física por la apertura que éstas brindan en comparación con las asignaturas básicas. Por ejemplo, una estudiante de tercer grado se manifestó en los siguientes términos: "Me gusta la clase de hogar. En ella pinto, recorto y hago recuerdos para el día de la madre y el padre". Es notorio que en este caso se aplica el pensamiento de Piaget quien aduce que el niño aprende jugando. Piaget (citado por Blanco y otras, 1999: 12) concibe a los niños como constructores de su conocimiento de ahí la importancia de clases con material concreto donde pueda manipular, crear, y descubrir por sus propios medios.

En el caso de las niñas de quinto año se sintieron muy bien en un ambiente que reforzó su esfuerzo mediante un reconocimiento público: "Me gusta cómṕuto porque hacemos proyectos. Investigamos y luego lo expusimos. Nos aplaudieron y nos sentimos muy contentas por lo bien que expusimos el tema" (pareja de alumnas de quinto). Consideran que 
las materias básicas no son lo suficientemente interesantes, debido a la cantidad de materia que escriben. Esto porque la metodología utilizada es pasiva, se usan mucho los libros de texto, fichas y pizarra, lo cual les provoca cansancio y pocos deseos de completar los trabajos. De lo anterior, es importante apuntar que en las materias básicas es necesario realizar un mayor esfuerzo en cuanto a trabajo con material concreto, dado que, como indican los estudiantes, los contenidos se abordan demasiados teóricos. Por lo tanto, desde el punto de vista de los estudiantes, los maestros de las materias básicas deben realizar mayores esfuerzos para innovar. Lo anterior, no implica que el maestro no innove, sino que la forma en que implementa el currículo no es lo suficientemente atractivo para el estudiante. Aquí se encuentra un primer punto al que se debe prestar atención cuando se implementan innovaciones educativas.

Aspecto contrario se presenta en las materias especiales, ya que por su naturaleza estas tienden a ser más prácticas que teóricas. Sin embargo, en estas materias, no por el hecho de que sean las preferidas por los estudiantes, se pueda concluir que se está innovando. En cuanto a las materias básicas los niños mostraron preferencia por las matemáticas, aunque no es la materia en que obtienen la calificación más alta. La describen como una materia práctica y aplicable a la vida cotidiana. Referente a las demás, concluyeron que son aburridas por la cantidad de teoría que éstas conllevan. Esto reafirma lo que se ha venido indicando acerca de la dificultad de los docentes para implementar el currículum de una manera amena y atractiva para los alumnos. Esta afirmación se respalda con las respuestas emitidas por doce niños quienes consideran que aprenden más con trabajos prácticos, esto lo dicen en los siguientes términos: "Me gustan las clases de Hogar, Cómputo e investigación en cómputo porque aplico lo que aprendo en la escuela. También Matemáticas la aplico al ir a la pulpería". 
Es fundamental que a los alumnos se les refuerce esa actitud positiva hacia las materias citadas, aprovechando que por su carácter práctico permite trabajar con material concreto. En el caso de las demás asignaturas básicas, el esfuerzo que se haga por ligarlas con la realidad cotidiana se verá recompensado con una imagen más positiva. Esto redundará en beneficio de los alumnos que efectivamente tendrán un aprendizaje más duradero y de sentido para ellos. En consecuencia, se debe revertir la opinión que tienen ellos de que las maestras no siempre les hablan sobre el éxito de sus aprendizajes. Para los alumnos, el que se exhiban sus trabajos es un elemento motivacional que va estrechamente ligado al ego de mostrar lo que ha hecho bien. Inclusive, cuando se hace algún proyecto, se expone y recibe el reconocimiento los estudiantes aducen sentirse bien. Inclusive, varios niños de quinto recuerdan cuando fueron con una innovación a Nicoya.

Lo que se ha venido comentando se refuerza con lo que expresan otros niños del carácter práctico de las materias y su preferencia hacia ellas. A Kiara también le gustan las matemáticas "porque es más práctico y no me gusta la teoría", sin embargo, "llevo mejores notas en Estudios Sociales y en Ciencias". En el caso de Fresia, estudiante de Quinto Grado, su materia preferida es Música, pero las demás materias no le disgustan ni le cuestan. El caso de Yoshua, estudiante de Segundo grado, es muy diferente, afirma que:

"no me gusta escribir ni me gusta la escuela. La maestra nunca hace juegos y a mí me gusta jugar. Me encanta cuando no hay clases. Me gusta ir a la escuela pero a jugar, lo que menos me gusta es escribir. Con mis compañeros me llevo más o menos por eso me gusta trabajar en grupo pero de amigos. Mis mejores notas son en Español y Ciencias. Las matemáticas no 
me gustan. Las tareas las hago solo en clases porque no recibo ayuda de mis papás."

A pesar de esta imagen tan negativa, y un poco rebelde, que este niño muestra hacia la escuela y el proceso educativo, la mayoría de los niños la valoran positivamente. La escuela es parte importante para ellos y muestran una actitud positiva de la estadía en ella. En primer lugar, afirman que no van por obligación, sino por el deseo de ser mejores y convertirse en buenos profesionales de gran ayuda para el país. Aseguran que el día que no asisten a clases extrañan la escuela, sus compañeros y su maestra:

"No deseo irme para otra escuela dado que me siento feliz con los compañeros. Vengo a la escuela con mucho gusto y me gusta por la mañana, aunque algunos compañeros llegan tardísimo. Me encanta escribir y cuando no vengo a la escuela me aburro ya que solo veo televisión o me mandan a hacer mandados" (Niña de III Grado).

Los niños manifiestan que prefieren asistir a clases en el período de la mañana. Afirman que sienten que les rinde más la tarde para jugar y estudiar. Un aspecto importante a rescatar es que, a criterio de los niños, sus papás tienen una imagen positiva de sus maestras. Consideran que ellas trabajan siempre o casi siempre bien. Esta percepción permite reforzar el proceso de aprendizaje de los niños al contar con la colaboración de los padres. El que los padres visualicen a las maestras de manera positiva puede reforzar el interés del niño por el aprendizaje. Los comentarios positivos hacen que los niños tengan mayor respeto y admiración hacia el docente. Ese respeto y admiración se puede traducir en mayor facilidad para implementar estrategias didácticas y elementos innovadores dentro del aula. 


\section{SIGNIFICADO DEL CONCEPTO DE INNOVA- CION EDUCATIVA EN LOS DOCENTES}

Un aspecto importante que se quiere rescatar en el presente estudio es el significado que, efectivamente, el maestro le asigna al concepto de innovación. De acuerdo con la forma cómo se conceptualice la innovación así será la implementación en el aula. Inclusive, lo que para un maestro pueda ser innovación, para otros colegas o estudiosos de la materia no lo es.

En el caso de los maestros entrevistados innovación es:

- Dar a conocer algo nuevo.

- Hacer cosas diferentes, logrando aprendizajes significativos a través de algo sencillo.

- Son formas que utiliza el docente para hacerse entender a los estudiantes.

La mayoría de los docentes coinciden en que innovar es cambiar la forma tradicional de hacer las cosas, por algo totalmente nuevo y sencillo, que permita el logro de aprendizajes significativos; entre otros conceptos se cita:

"Para mí, innovar son las formas diferentes en la que el docente es capaz de trasmitir al estudiante sus conocimientos, ya sea oral, práctico o escrito y todas las maneras de hacerse entender mediante estrategias nuevas" (Docente).

Como parte de este trabajo, también se intentó conocer si el plan de clases incorporaba las innovaciones educativas. Al respecto, una docente contestó que sí, porque se dan como parte del proceso de desarrollo de los planes de 
grado que imparte. Además, argumenta que facilita la labor de enseñanza y produce clases dinámicas y participativas. La mayoría de los docentes afirman que tratan de incorporarlas, pero no siempre se puede. Aducen falta de tiempo, exceso de contenidos, escasez de presupuesto y el trabajo de doble jornada. Es muy agotador para el docente estar en el centro educativo de 7:00 a 5:15 P.M, tomando en cuenta que, la gran mayoría, son viajantes. Esta situación contradice lo que ellas mismas habían afirmado anteriormente.

Para Juana (nombre ficticio) innovar es una tarea un poco difícil porque según ella:

"La doble jornada, la cantidad de contenidos, el poco presupuesto con que se cuenta y la presión del sistema con fechas establecidas, hace que yo no pueda realizar o cumplir con la difícil tarea de innovar.

No siempre el no innovar recae en el docente porque en el aula encontramos alumnos muy negativos que no quieren participar y también uno conoce el grupo que siempre le gusta hacerlo".

Lo anterior es necesario destacarlo porque, generalmente, los docentes se guían por los programas de estudio brindados por el Ministerio de Educación (MEP), que son bastante rígidos y muchas veces no permiten espacios para formas creativas. Sin embargo, cuando el docente es creatiwo encuentra el espacio propicio para innovar. La experiencia del docente en el ejercicio de su profesión es fundamenral a la par del interés y la habilidad para manejar diferentes escenarios

En cuanto al proceso de sistematización es igualmente dificil de realicarlo, según las docentes, debido a la falta de tiempo y de recursos. Solamente cuando se debe asistir a la Feria de Innovaciones Educativas es cuando se intenta 
sistematizar las experiencias. Las educadoras consultadas coinciden con lo que apunta Ana Lorena Vargas (2003, p.1), en el sentido de que en general los docentes quieren sistematizar las experiencias pero que, no obstante, son pocas las personas que lo logran. Para Vargas (2003, p.1) esto se da por tres razones: "1.Es una tarea compleja y especializada. 2. No se cuenta con definiciones claras sobre qué sistematizar y quiénes deben hacerlo.3. En la práctica no se le da prioridad: institucionalmente no se le asigna tiempo." Por su parte, las docentes de la Escuela Presbítero José Daniel Carmona aducen que sienten que no es una prioridad institucional, ni para las altas autoridades de la Dirección Regional de Enseñanza de Nicoya, ni para la supervisión del circuito. Los espacios para la sistematización de experiencias no existen, menos para compartir esas experiencias. Desde este punto de vista, sienten que lo que más les interesa es que se cumpla con la rutina que va absorbiendo poco a poco a los docentes más dinámicos, quienes terminan decepcionados del sistema.

La capacitación también es otro elemento de vital importancia pues a pesar del interés por aplicar los procesos de innovación no se cuenta con el instrumental suficiente para ello. Debido a lo anterior se le planteó a las docentes que si habían recibido alguna capacitación para planear, tomando en cuenta las innovaciones educativas, destacaron que no; todas las capacitaciones las dio el SIMED cuando inició su plan piloto con su programa de Escuelas Líderes; sin embargo, hubo problemas, a veces logísticos o de convocatoria. Además, se debe destacar que mucho del personal docente rotó y no estaba en la escuela para la época en que se implementó el programa del SIMED. Esto dificultó la aplicación del conocimiento en el aula escolar. Por lo tanto, lo que han aprendido es por medio del intercambio de experiencias informales con otros compañeros, y lo hacen 
por ayudar a los alumnos. Al respecto, María (nombre ficticio) se refiere a este tema en los siguientes términos:

"Yo solo he recibido algunas charlas, pero no me llenan. A veces algunas personas con experiencia me dan algún consejo que logra llenar mis expectativas y me ayudan mucho más que pasar horas escuchando alguna capacitación dada por la Dirección Regional"

En cuanto a este punto, hay diversas opiniones, pues la teoría con la práctica debe estar muy relacionada, y en cuanto a innovaciones esto no se está dando debido a varios factores: no se le da el seguimiento debido a las experiencias docentes; en otros casos no se sistematizó el trabajo realizado y no quedó constancia del mismo. También consideran que no se tuvo éxito por no haber llegado a todas las escuelas, solo a las Líderes. Aún así, no todo fue negativo porque también se lograron nuevas experiencias y hasta cierto punto se sistematizaron unas pocas.

Otra debilidad encontrada se relaciona con que a muchos docentes no les gusta leer y eso influye directamente con la práctica. A pesar de que en la escuela objeto de estudio se encuentra todo el acervo bibliográfico del Proyecto SIMED, únicamente se consulta cuando al docente se le deja un trabajo en la Universidad; de lo contrario, este material permanece sin uso. En este sentido, es importante realizar círculos de estudios en el ámbito institucional y auto capacitaciones que permitan un mejoramiento de la práctica pedagógica diaria. Lo anterior, es muy importante para mejorar a nivel micro, es decir, en el ámbito de la escuela, la calidad de la enseñanza. Todo esto conlleva beneficios para los estudiantes y los docentes. 


\section{ELEMENTOS OBSERVADOS EN EL PLANEAMIEN. TO Y EL DESARROLLO DE LA LECCIÓN}

El estudio en cuestión también comprendía la revisión del plan de clases. Se pudo detectar que de los cuatro diarios de clase, en dos de ellos estaban concatenando muy bien los objetivos, contenidos, procedimientos, valores y evaluación; sin embargo, en los otros dos, los objetivos no estaban acordes con los procedimientos, ya que para lograr el objetivo planteado debían aumentar la cantidad de experiencias de aprendizaje. Muchos de esos objetivos eran más amplios que los procedimientos, lo cual dificulta su logro. Por lo tanto, se deduce que es necesaria una mayor capacitación en este campo. Cabe manifestar también que algunos de estos docentes limitan su labor escolar a trabajar con manuales, libros de textos de la colección Santillana u otros. Los alumnos logran un trabajo muy individualizado y no se destina tiempo para el intercambio de experiencias a través de diversas técnicas como: plenarias, mesas redondas u otras que fomenten discusión ante los diferentes puntos de vista y se logre así una buena reflexión hacia ciertos acontecimientos que imperan en la realidad cotidiana.

En el caso de la docente de segundo grado, se pudo observar su interés porque los alumnos completaran el resumen escrito, obviando los comentarios y reflexiones; debe aclararse que este nivel exige mucha atención y dedicación por parte del docente. Es importante destacar que existen muchos distractores que inciden en la concentración de los niños, debido a que el salón de actos está junto a su aula, así como esta se ubican cinco aulas más en el mismo pabellón, una enfrente de otra, por lo que los ruidos se entrecruzan; además, esta aula está contigua a los baños, lo que termina de afectar el aprendizaje de los niños. Todos los factores antes mencionados pueden influir en el rendimiento del 
niño así como en la efectividad de las clases que la docente imparte.

En tercer grado cabe mencionar que la docente organizó una especie de plenaria para extraer las experiencias previas de sus alumnos acerca del tema, se dedujeron las conclusiones por parte de ella y escribió un resumen en la pizarra para que los niños lo copiaran. Se observó que las condiciones del aula no eran apropiadas para el uso de la pizarra porque no existía suficiente luz y a algunos alumnos se les dificultaba observar correctamente. Además, la puerta se mantenía cerrada para atenuar un poco los distractores arriba mencionados, esta situación se convierte en un factor negativo para los niños y la práctica docente.

En cuanto a la docente de sexto grado, se observó que utiliza una metodología muy activa y variada. Sus alumnos intercambian experiencias constantemente a través de diferentes técnicas metodológicas como trabajos grupales e individuales. Esto facilita un aprendizaje más dinámico y horizontal provocando mayor participación y efectividad en la construcción de los conocimientos.

Es importante mencionar que todas las docentes cuentan con su planeamiento al día, además, una decoración atractiva del salón de clase, lo que propicia un mejor ambiente escolar. Este detalle es digno de rescatar dado lo limitado de los recursos con que se cuenta para este fin. Todos los planeamientos contemplan motivación al inicio de la jornada, esto con el propósito de preparar al estudiante positivamente con respecto al tema que se abordará. Entre los materiales más utilizados en el desarrollo de las lecciones están: libros, fichas, fotocopias pizarra y en pocos casos material audiovisual.

Referente a la coherencia entre el planeamiento y la lección es evidente, pero en algunos casos se aprecia más que en otros. Esto parece ser más sustancial para los grados 
superiores ya que los alumnos tienen mayor grado de madurez, lo que facilita la criticidad, la discusión y el análisis de las temáticas.

\section{RECURSOS DIDACTICOS Y LA INNOVACIÓN EN LA ESCUELA PRESBITERO JOSE DANIEL CAR. MONA}

En cuanto a los recursos didácticos, a los docentes se les plantearon las siguientes interrogantes: ¿Considera usted que los recursos didácticos que utiliza son innovadores? ¿Por qué? al respecto consideran que unos recursos sí son innovadores, otros no. Lo que les da esta condición es la forma como se utilizan. Los que construyen los niños sí son innovadores porque contemplan sus conocimientos, necesidades y generan el aprendizaje que se busca lograr en ellos.

Considerando que muchos recursos didácticos utilizados en el aula los realizan los mismos niños, surgió la interrogante sí la docente solicitaba ayuda a los padres de familia en la elaboración de los recursos didácticos utilizados. $\mathrm{Al}$ respecto, se logró determinar que la ayuda se solicita a los padres de familia en ciertas ocasiones, por ejemplo, cuando se requieren materiales y no se tienen las comodidades o implementos necesarios en el aula para elaborarlos. Dado lo anterior es importante conocer los aspectos que el docente toma en cuenta al seleccionar los recursos didácticos utilizados en el aula.

Lo primero que el docente toma en cuenta es que despierten el interés en los niños para lograr un aprendizaje más duradero. Luego, que permita lograr el objetivo Y también, otro muy importante, es el factor económico. Se trata de utilizar material de bajo costo que esté al alcance de todos.

Lo anterior implica un delicado planeamiento de la innovación por implementar con determinados materiales 
contextualizados del entorno porque si su costo es alto, esta podría fracasar y se perdería todo el trabajo realizado. Sin embargo, lo más importante de las innovaciones educativas es el impacto que pueda tener sobre el aprendizaje de los niños. Al respecto, los docentes afirman que se da un aprendizaje significativo de los niños ya que estos vivencian el proceso. Si el alumno es capaz de explicar lo que vivió, el aprendizaje es entonces perdurable. Además, si la innovación facilita el conocimiento o la forma de adquirirlo, no se convierte en algo repetitivo, y lo más importante, los niños lo pueden aplicar a su vida diaria.

En cuanto a la forma de evaluar el aprendizaje de los alumnos los docentes apuntaron que hay muchas formas de hacerlo, y entre ellas:

- Se utilizan diversas técnicas como por ejemplo: juegos, dibujos, mesas redondas, plenarias, interrogantes.

- Hay participación individual y grupal (discusiones, plenarias, phillips 66)

- Además se recurren a las pruebas escritas.

- Formativa: insertar dentro del plan diario de cada asignatura diferentes valores morales, espirituales, cívicos.

En este sentido, el docente debe recurrir a formas más creativas de evaluación en vista que el sistema actual implica pruebas que no permiten la creatividad. En muchos casos, se pone mayor énfasis en una respuesta final que a la creatividad del niño durante el proceso y a la forma a que se llegó al resultado. Teoría como la de la evaluación auténtica es más pertinente en el contexto del constructivismo y no las formas bastante inflexibles que se utilizan actualmente.

Otras de las interrogantes planteadas fue la siguiente: ¿Considera usted que las estrategias metodológicas 
utilizadas en el aula estimula la participación de sus estudiantes? La respuesta es que sí estimula la participación, pero no en un $100 \%$. No se puede obviar que muchos estudiantes son más abiertos que otros, con mejor razonamiento y con más interés por el estudio. Algunos estudiantes contestan con monosílabos y no argumentan sus opiniones. Sin embargo, la experiencia ha demostrado que aquellas clases dinámicas, menos rutinarias, e innovadoras propicia una mayor participación del niño. Según la opinión de una docente consultada: "Las estrategias estimulan la participación en el aula solo en parte ya que siempre son los mismos los que participan y hay un sector vago que no muestra interés por las actividades realizadas en la clase". De ahí que, a criterio de las docentes, no se pueda estimular al 100\% de la población estudiantil.

\section{CONCEPTO DE INNOVACIÓN DESDE LA PERS. PECTIVA DEL DIRECTOR}

La experiencia del director en este aspecto es importante debido a que brinda información relevante acerca de la visión del administrador educativo, en torno a las innovaciones. Muchas veces, el fracaso de una innovación nace a partir del poco apoyo de los superiores jerárquicos hacia los docentes. En cuanto al concepto de innovación afirma que innovar es "Cambiar la forma tradicional de hacer las cosas partiendo de un problema detectado en el aula, poner en práctica algún plan remedial que sea simple y que a los niños les permita salir adelante de una manera efectiva y que este aprendizaje sea duradero". Es importante indicar que el director maneja un concepto más práctico de la innovación ya que para él debe ser simple, pero efectivo y duradero. Además, señala que el docente es el encargado de plasmarlo en su plan de trabajo. 
En cuanto a que si todos los docentes de su institución innovan respondió que se dan casos, pero no todos porque algunos sí les gusta salir de lo tradicional, y por lo tanto, considera que cuenta con los dos tipos de docentes: los tradicionales y los que tratan de salirse de este esquema. Afirma que él no obliga a los docentes a innovar y tampoco lo contempla en el plan anual porque el sistema no está para eso, se exige cumplir con programas y contenidos, y si éstos se dedican a hacer muchas actividades dejarían de lado otras que también se deben atender.

Demostró estar enterado de las innovaciones que se realizan en su escuela y explicó que le gusta más que sea voluntario y no que el personal lo sienta como una orden. Esto podría llevar a perder el interés y a administrar la rutina, con esto se puede interpretar de que él no motiva a su personal, porque lo deja a juicio de cada uno; además, son pocos los espacios disponibles para sacar el personal con frecuencia, debido a los doscientos días lectivos que se deben cumplir, y por ello, el MEP no tiene apertura para eso. También considera que los gobiernos no atacan el mal y en cambio hacen otras cosas, por lo que deberían buscar estrategias para comprometer al docente a ser mejor.

Por otra parte, las Direcciones Regionales asumen una posición muy tibia hacia este tema. No brindan ningún tipo de asesoría provechosa para el docente, y por ende, para el trabajo de aula. Es en los días más cercanos a la Feria de Innovaciones Educativas cuando éstas se pronuncian, sin saber si ha habido un verdadero proceso. Lo único que interesa es tener un número de escuelas participantes; sin haberles brindado ningún tipo de asesoría ni recursos económicos para solventar los gastos. De ahí que la mayoría de educadores se sientan desmotivados y un poco abrumados cuando se les exige lo que no se da. 


\section{EL ROL DE LA DIRECCIÓN EN EL PROCESO DE INNOVACION}

Por último, se quería indagar acerca del apoyo que como director le brindaba al docente innovador. En este sentido indicó los siguientes aspectos:

- Darle plena libertad para que su labor en el aula sea totalmente innovadora y ponga en práctica su capacidad y creatividad profesional.

- Inculcarles hacer uso del acervo bibliográfico que para tal efecto se encuentra en la biblioteca del SIMED, la cual está disponible en la institución.

Apunta que muchos docentes, lamentablemente, no se dan a la tarea de innovar, argumentan el factor tiempo; sin embargo lo que no quieren son complicarse y prefieren la rutina, otros implementan actividades muy creativas y no las sistematizan en su plan diario, entonces se pierde la labor que realizan en el aula. Finalmente agrega que el docente preocupado por innovar es aquel que se siente comprometido con su labor y tiene bien claro los objetivos que desea lograr, y que en la actualidad estos docentes son pocos.

\section{COMPARACIÓN DE LA INFORMACIÓN PRO. PORCIONADA POR ALUMNOS, DOCENTES Y DIRECTOR}

Con el propósito de realizar una triangulación de la información se hace una comparación de los resultados de acuerdo con la fuente que la emitió: alumnos, maestras y director.

En el caso del presente estudio, se utiliza una triangulación de datos, que confronta la información brindada por 
los tres principales elementos componentes de la vida académica de una institución de enseñanza como son los niños, las maestras y el director. Para una mayor claridad de los aspectos por evaluar se presenta la siguiente comparación:

\section{CRITERIOS COMPARATIVOS ACERCA DE LA INNO. VACIÓN DESDE LA PERSPECTIVA DE LOS NIÑOS, LAS MAESTRAS Y DIRECTOR}

\begin{tabular}{|c|c|c|c|}
\hline ASPECTO & NIÑOS & MAESTRAS & DIRECTOR \\
\hline $\begin{array}{l}\text { Significado de in- } \\
\text { novación. }\end{array}$ & $\begin{array}{l}\text { - Aprender jugan- } \\
\text { do. Hacer o crear } \\
\text { cosas. Aprovechar } \\
\text { materiales viejos } \\
\text { para hacer otros } \\
\text { trabajos. }\end{array}$ & $\begin{array}{l}\text { - Dar a conocer } \\
\text { algo nuevo; hacer } \\
\text { cosas diferentes, } \\
\text { logrando aprendi- } \\
\text { zajes significativos } \\
\text { a través de algo } \\
\text { sencillos. Son for- } \\
\text { mas que uriliza el } \\
\text { docente para ha- } \\
\text { cerse entender a } \\
\text { los estudiantes. }\end{array}$ & $\begin{array}{l}\text { - Poner en práctica } \\
\text { plan remedial par- } \\
\text { tiendo de un pro- } \\
\text { blema en el aula. } \\
\text { Que sea simple. } \\
\text { Que permita un } \\
\text { aprendizaje dura- } \\
\text { dero. }\end{array}$ \\
\hline $\begin{array}{l}\text { Opinión en torno } \\
\text { al planeamiento } \\
\text { didáctico. }\end{array}$ & $\begin{array}{l}\text {-Las clases son bas- } \\
\text { tante aburridas. } \\
\text { - Algunos aducen } \\
\text { que les gustan to- } \\
\text { das las materias } \\
\text { pero dan preferen- } \\
\text { cia a algunas espe- } \\
\text { ciales. Las mate- } \\
\text { rias son demasiado } \\
\text { teóricas. Les gusta } \\
\text { materias prácticas } \\
\text { donde se hagan } \\
\text { cosas como pintar. }\end{array}$ & $\begin{array}{l}\text { - Consideran que sí } \\
\text { incorporan ele- } \\
\text { mentos innovado- } \\
\text { res dentro del pla- } \\
\text { neamiento. } \\
\text { - Incorporar ele- } \\
\text { mentos innovado- } \\
\text { res produce clases } \\
\text { dinámicas y parti- } \\
\text { cipativas. }\end{array}$ & $\begin{array}{l}\text {-El docente debe } \\
\text { contextualizar los } \\
\text { contenidos utili- } \\
\text { zando el entorno } \\
\text { comunal y desde } \\
\text { esta perspectiva } \\
\text { debe innovar. } \\
\text { - Le da libertad al } \\
\text { docente para in- } \\
\text { novare incorporar } \\
\text { esta dentro del } \\
\text { planeamiento. }\end{array}$ \\
\hline $\begin{array}{l}\text { Rol de la dirección } \\
\text { - innovación }\end{array}$ & & $\begin{array}{l}\text {-No es prioridad } \\
\text { institucional. } \\
\text { - No se le asigna } \\
\text { recursos ni tiempo } \\
\text { para la sistematiza- } \\
\text { ción ni para com- } \\
\text { partir experien- } \\
\text { cias. }\end{array}$ & $\begin{array}{l}\text {-Trata de que uti- } \\
\text { lice el material bi- } \\
\text { bliográfico del SI- } \\
\text { MED. } \\
\text { - Le da libertad al } \\
\text { docente para in- } \\
\text { novar. } \\
\text { - No contempla las } \\
\text { innovaciones en } \\
\text { el plan anual. }\end{array}$ \\
\hline
\end{tabular}


Realizando primeramente una comparación por constructo es posible visualizar, de forma conjunta, las diferentes percepciones que tiene cada uno de los actores investigados. En primera instancia se analizará el significado de innovación para los niños, las maestras y el director. La importancia de lo anterior estriba en poder efectivamente verificar qué es lo que cada uno entiende por innovación. En el caso de los niños perciben innovar como aprender jugando y aprovechar materiales viejos para hacer otros trabajos. Sin embargo, para las maestras es dar a conocer actividades y resultados nuevos; hacer cosas diferentes, logrando aprendizajes significativos a través de cambios sencillos. Desde esta perspectiva, maestros y niños difieren en su concepción. De ahí que una de las quejas de los estudiantes sea que las materias básicas son demasiadas teóricas. Para los niños innovar es que su aprendizaje sea lo más natural posible; es decir, que se aprenda jugando; que se hagan o se creen productos concretos coincidiendo esto con la teoría constructivita de Piaget.

Para las maestras es dar a conocer algo nuevo sin especificar de qué forma. Tiende más a lo teórico que a lo práctico, más a lo abstracto que a lo concreto. Por lo tanto, es normal entonces que el estudiante aduzca demasiada teoricidad en el abordaje de los contenidos de las materias básicas. Además, para las maestras innovar es hacer cosas diferentes logrando aprendizajes significativos a través de algo sencillo. Eso, quizás, pueda coincidir con lo que apuntan los niños: innovar es aprovechar materiales viejos para hacer otros trabajos.

En el caso del director plantea que innovar es poner en práctica un plan remedial partiendo de un problema en el aula, debe ser simple (la innovación), y que permita un aprendizaje duradero. Un plan remedial no necesariamente es una innovación. Pero sí se está de acuerdo con el director en 
el sentido de que ésta debe ser simple y permitir un aprendizaje duradero. En esto coinciden las maestras y el director, donde apuntan que con la innovación se deben lograr aprendizajes significativos a través de acciones sencillas.

Sintetizando este primer constructo se puede anotar que innovación educativa, desde la perspectiva de los educandos, las maestras y el director es poner en práctica un plan que parta de una necesidad diagnosticada en el aula para producir aprendizajes significativos y duraderos. Además, debe cumplir con las siguientes características: ser simple, agradable a los niños, permitir un aprendizaje duradero, en la medida de lo posible utilice material concreto: y por último, que sea más práctico y menos teórico.

Por lo tanto, esto es lo que vivencian los diferentes actores. Y desde ese ángulo es donde se debe realizar el esfuerzo en el ámbito de la escuela y el aula para implementar exitosamente innovaciones educativas. Además, es fundamental crear una cultura de sistematización de las experiencias para compartirlas con otros compañeros y escuelas. Compartir estas experiencias podría llevar a la búsqueda conjunta de soluciones a problemas similares, así como a la teorización en beneficio de la educación y de los niños.

El problema fundamental por el cual no se sistematiza está, en realidad, en la falta de capacitación e información, sobre cómo hacerlo. Muchas de las sistematizaciones se quedan en la narración de la experiencia y descripción del proceso; cuando verdaderamente una sistematización es mucho más que eso. Esta lleva una serie de pasos ordenados como son los siguientes (Jara, 1994, citado por Vargas): describirlas, problematizarlas, traducirlas, interpretarlas, comprenderlas, proponer las acciones y actuar. En otras palabras, es necesario actuar urgentemente en ese sentido.

En cuanto a la opinión que tienen los alumnos, las maestras y el director con respecto al planeamiento didáctico y si 
dentro de él se incorporan elementos innovadores, las versiones son variadas y divergentes. De las respuestas emitidas por los niños se deduce que el planeamiento didáctico no contiene elementos innovadores que los motive. Apun$\tan$ que las clases son bastante aburridas lo que conlleva a decir que la labor de aula no se está planificando para hacerla agradable. Además, manifiestan que las materias son demasiados teóricas y que su preferencia son por las materias prácticas como Cómputo y las clases de Hogar. En otras palabras, ellos prefieren las clases que involucre la práctica tales como pintar, realizar proyectos y trabajar con material concreto.

Por el contrario, las maestras piensan que sí incorporan elementos innovadores dentro del planeamiento y lo plasman en el aula. Aducen que esos elementos innovadores crean clases dinámicas y participativas. Sin embargo, se debe tomar en cuenta la opinión de los niños y revisar lo que se está llevando al aula y evaluar si efectivamente es una innovación. Lo anterior es importante porque, tal como aducen las mismas maestras y el director, se trata de que se dé un aprendizaje duradero y significativo, y si los niños no están percibiendo las clases como agradables, dinámicas y motivantes, está fallando algún aspecto de la innovación o no se está innovando.

En los planeamientos observados se rescata el intento por innovar, pero aún es insuficiente como para afirmar que se dan elementos totalmente innovadores. Las razones, ya se han anotado con anterioridad, se rescatan con más detalle en las conclusiones.

En el caso del director, apunta que da la plena libertad al docente para incorporar dentro del planeamiento las innovaciones. Es del criterio de que el docente debe contextualizar los contenidos utilizando elementos innovadores extraídos del entorno comunal. Desde esa perspectiva 
se debe innovar. Sin embargo, dice ser consciente, que muchos docentes prefieren no complicarse y realizan su trabajo de manera rutinaria.

El último aspecto que interesa rescatar en esta comparación que se viene realizando es el papel de la dirección con respecto al proceso de innovación. Los teóricos de la administración (Villegas, 1994, p.56, entre otros) son claros al afirmar que el liderazgo en una organización es fundamental para marcar rumbos; de ahí que la dirección deba ejercer un papel activo, de guía, de motivador, de comunicar los propósitos y las metas de la organización; debe poseer capacidad para organizar, capacidad ejecutiva y saber delegar funciones y autoridad adecuadamente. En cuanto a las funciones de la dirección con respecto a las innovaciones educativas las maestras perciben diversos elementos. Uno de ellos es que las innovaciones no son una prioridad institucional, e inclusive, no es prioridad ni para la supervisión ni para la misma Dirección Regional de Enseñanza de Nicoya, del Ministerio de Educación Pública. Lo anterior por cuanto no están incluidas ni siquiera en el Plan Institucional. Otro aspecto, que apuntan, es que no se le asigna recursos ni tiempo para la sistematización, ni para compartir experiencias en el ámbito institucional y muchos menos a nivel de circuito. Es decir, todos aquellos elementos innovadores que se puedan estar llevando al aula quedan en el anonimato, con el perjuicio para los niños como para los mismos maestros. Compartir sistematizaciones es fundamental para mejorar el quehacer diario en el aula, y por ende, la calidad de la educación que reciben los niños.

Por su parte, el director arguye que él da libertad al docente para innovar. Este punto parece motivador; sin embargo, de acuerdo con lo expresado por los docentes, no existe una cultura institucional que permita que esa libertad se traduzca en propuestas concretas, por lo que esta cultura debe 
ser impulsada desde la dirección. Una vez establecida, es mucho más fácil que se produzcan propuestas que se apliquen y se sistematicen. Un punto más que añade el director es que él trata de incentivar que se utilice el material bibliográfico del SIMED sobre la temática. Pero, los maestros no tienen el hábito de lectura. Además de eso, la ausencia de capacitaciones efectivas no permite que se despierte interés por la temática.

\section{CONCLUSIONES}

1- Se concluye que las docentes de las materias básicas tienen un concepto de innovación bastante claro dado que estas lo conceptualizan como:

- Dar a conocer algo nuevo mediante estrategias sencillas.

- Salirse de la rutina.

- Hacer cosas diferentes, logrando aprendizajes significativos a través de algo sencillo.

- Son formas que utiliza el docente para hacerse entender de los estudiantes.

Este significado coincide con los criterios propuestos por Collorete (citado por Murillo y otros, 1996) quien conceptualiza la innovación como "la acción de encontrar, descubrir o inventar algo nuevo en relación con un entorno dado a algún problema".

Sin embargo, los alumnos entrevistados no manejan claramente el concepto de innovación, como tal, pero si aducen haber participado y gustarle las actividades realizadas. Consideran que se aprende más, se sale de la rutina y se desarrolla mucha participación por parte de sus compañeros. Este factor incide en la opinión de los niños sobre las 
materias especiales, ya que se sienten motivados, al realizar actividades prácticas como pintar, jugar, recortar. Por otra parte, argumentan que las materias básicas son muy teóricas y hay que escribir mucho, por lo que se cansan y se aburren, Es por eso que se recomienda realizar un esfuerzo mayor para trabajar los diferentes contenidos con material concreto.

2-Se identificaron los siguientes factores que pueden afectar la labor de innovar:

Tiempo: Las docentes entrevistadas, en su mayoría, son viajantes y esto implica un horario de 6:00 AM a 7:00 PM, lo que obstruye la elaboración de materiales concretos para trabajar en clase. Si a eso se le suman los trabajos extraclases, registros, notas, unidades y actividades extracurriculares que realizan en sus hogares, el tiempo disponible es todavía menor; reconocen que tratan de innovar, pero se sienten limitadas por este factor. Por lo tanto, se recomienda hacer círculos de estudios, entre el personal, para compartir experiencias y estrategias metodológicas puestas en práctica que hayan dado resultados.

Presupuesto: No se recibe ningún aporte económico por parte del MEP o la institución. No hay ningún rubro para ello; se cuenta únicamente con una cuota fijada por los padres de familia cada en forma mensual y que no todos pagan. Esto limita al docente a disponer de recursos. Dicha cuota abastece hasta los materiales utilizados en las materias especiales.

Cansancio (desgaste físico y emocional): La doble jornada, la revisión de diferentes trabajos (tareas), el planeamiento didáctico diario, las actividades extracurriculares y otras más, hacen que el docente viva un estrés y un desgaste permanente. Lo anterior quedó de manifiesto en 
las diferentes entrevistas realizadas a las docentes quienes destacan que todos estos factores inciden negativamente en su labor de aula. Sin embargo, la mayoría de los docentes, aceptan y buscan una doble jornada, porque los niveles salariales que se perciben en la educación primaria son poco competitivos en relación con otras profesiones. Por lo tanto, a pesar del cansancio que esto implica es preferible a una jornada sin alterno.

3. Dificultades para la sistematización de experiencias. Las educadoras aducen que la poca capacitación sobre este tema, por parte del MEP, y la falta de tiempo son limitantes que obstaculizan la sistematización de experiencias. Hay que recalcar que la sistematización se convierte en un instrumento que permite, a los docentes, crear un banco de datos sobre las diferentes metodologías desarrolladas en el salón de clase; al compartirlas con otros docentes, permite enriquecer el quehacer educativo. Este es un proceso que puede ser confrontado con otras experiencias de una manera flexible, donde lo más importante, es obtener nuevas experiencias que servirán de base para nuevos conocimientos.

4- Importancia de las innovaciones en el salón de clases. El arte de innovar en el salón de clases, fomenta y desarrolla valores y actividades positivas. Las innovaciones permiten la participación de estudiantes en la planificación de la misma. Esto le ofrece un ambiente de libertad, de pensamiento y sentimiento, además les brinda confianza haciéndoles sentir que cada uno es importante para la maestra. Estas actitudes, o grado de empatía, del alumno hacia su maestra propician un ambiente adecuado para desarrollar tareas innovadoras.

5- La innovación depende de las características individuales del docente. Cada educador pretende lograr 
aprendizajes significativos en el aula para mejorar la calidad de la educación. Partiendo de esa premisa, el director considera que él no debe imponer la labor innovadora a ningún docente; y por eso, no es contemplado en el plan anual institucional, sin embargo, se manifiesta satisfecho con quien lo hace y le brinda el apoyo necesario para tal efecto.

6- El horario limita la posibilidad de innovar en el aula. Otra situación detectada son los horarios diseñado por la escuela en cada jornada al extremo que a veces no permite conservar la secuencia en las lecciones.

\section{Referencias bibliográficas}

Blanco, S y otras (1999). La Articulación entre el ciclo de transición y el primer año de la Educación General Básica. San José: Costa Rica, PROMECE-MEP.

Libedinsky, M (2001). La Innovación en la Enseñanza: Diseño y Documentación de Experiencias de Aula. Buenos Aires Argentina, Editorial Piados.

Murillo, E y otros (1996). Innovaciones Técnico-pedagógicas para una mejor calidad de la educación. San José, Costa Rica: SIMED.

Pérez, R y otros (2000). Hacia una práctica pedagógica constructivista. San José, Costa Rica: PROMECE.

Vargas, A (2003). La Sistematización de las Experiencias Educativas. División de Educación Básica, CIDE-UNA.

Villegas, J.J (1994). "La Dirección de los Centros Educativos desde una Perspectiva Micropolítica". En Revista de Educación. UCR. N 18 , Costa Rica. 
Entrevistas, observación directa e historia de vida

Docentes de la Escuela Presbítero José Daniel Carmona Briceño. Nandayure, Guanacaste. Octubre, 2003.

Director de la Escuela Presbítero José Daniel Carmona Briceño.

Nandayure, Guanacaste. Octubre, 2003.

Ocho niños de I ciclo de la Escuela Presbítero José Daniel Carmona Briceño. Setiembre del 2003.

Ocho niños de II ciclo de la Escuela Presbítero José Daniel Carmona Briceño. Nandayure. Setiembre, 2003. 